\title{
Clinical Characteristics and Prognosis of Rare Histological Variants of Bladder Cancer: A Single-Center Retrospective Study from China
}

This article was published in the following Dove Press journal: Cancer Management and Research

\author{
Dengxiong $\mathrm{Li} \mathbb{D}^{*}$ \\ Ao $\mathrm{Li}^{*}$ \\ Yubo Yang \\ Dechao Feng (D) \\ Facai Zhang \\ Xiaoming Wang \\ Yunjin Bai (D) \\ Yin Tang \\ Ping Han (D)
}

Department of Urology, Institute of Urology, West China Hospital, Sichuan University, Chengdu, Sichuan Province 61004I, People's Republic of China

*These authors contributed equally to this work
Correspondence: Ping Han

Department of Urology, Institute of Urology, West China Hospital, Sichuan University, Guoxue Xiang \#37, Chengdu, Sichuan Province 61004I, People's Republic of China

Tel +86-28-85422444

Fax +86-28-8542245I

Email hanpingwch@I63.com
Objective: To investigate the clinical characteristics and prognosis of patients with rare histological variants of bladder cancer (RHV-BC) in China.

Materials and Methods: Patients diagnosed as bladder carcinoma with RHV in our center, from March 2009 and April 2019, were included. The univariate and multivariate COX regression model were used to evaluate the association between clinical characteristics and overall survival (OS).

Results: A total of 54 (1.4\%) patients with RVH-BC were identified from 3803 potential patients with bladder cancer. The RHV classifications included micropapillary variant (MPV), sarcomatoid variant (SAV), neuroendocrine variant (NEV), nested variant (NV), plasmacytoid variant (PCV), and lymphoepithelioma-like variant (LEV), which were found in 19, 18, seven, six, three, and one patient, respectively. The mean of recurrence-free survival (RFS), cancer-specific survival (CSS), and OS of patients were 18.8 months, 37.0 months and 36.0 months, respectively. The multivariable analyses indicated that metastasis and $T \geq 2$ were independent risk factors of OS. Besides, 84.4\% (27/32) of patients who were receiving intravesical therapies (IVT) suffered recurrence. Patients with adjuvant chemotherapy (AC) had a recurrence rate of $64.7 \%$ (11/17).

Conclusion: $\mathrm{T} \geq 2$ and metastasis were independent risk factors of OS in patients with RHVBC. Considering a high recurrence following transurethral resection of bladder tumor (TURBT) and IVT, early radical cystectomy (RC) might be performed for patients with RHV-BC.

Keywords: bladder cancer, histologic variants, prognosis, overall survival

\section{Introduction}

Bladder cancer is the second most common urologic malignance, the prognosis has been improved over the past decades. ${ }^{1}$ However, it was found that patients with histological variants (HV) of bladder cancer had a worse prognosis than those

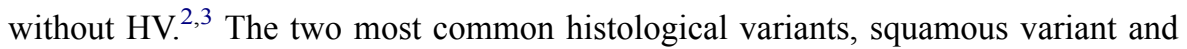
glandular variant, with a proportion of approximately $20 \%$ in all bladder cancer cases, have been discussed widely. ${ }^{4}$ But there were few studies focusing on other variants with much lower incidence, including micropapillary variant (MPV), sarcomatoid variant (SAV), neuroendocrine variant (NEV), nested variant (NV), plasmacytoid variant (PCV), and lymphoepithelioma-like variant (LEV), which were collectively named as rare histological variants of bladder cancer (RHV-BC) here. 
Since the World Health Organization (WHO) updated the classifications of urothelial carcinoma in 2016 to highlight the importance of an accurate diagnosis of bladder cancer, the proportion of $\mathrm{HV}$ in bladder cancer has obviously increased. ${ }^{4,5}$ Recently, HV was found to be generally associated with worse outcomes and more often upstaged after radical cystectomy (RC) than those without $\mathrm{HV}^{2,6}$ Intravesical therapies (IVT), including bacillus Calmette-Guérin (BCG) and chemotherapeutic drugs, have been proved effective to prevent or prolong the recurrence in patients with non-muscle invasive bladder cancer (NMIBC) without HV. ${ }^{7,8}$ However, some studies reported that patients with MPV receiving IVT had a worse outcome than early RC. ${ }^{9}$ Sui et $\mathrm{al}^{3,10}$ reported that neither neoadjuvant chemotherapy (NAC) nor adjuvant chemotherapy (AC) improved the overall survival (OS) of patients with MPV or SAV. On the other hand, several studies investigated the prognostic factors of RHV-BC. A higher proportion of locally advanced disease and lymphovascular invasion (LVI) were associated with MPVBC. ${ }^{11,12}$ However, few studies have paid attention to other subtypes of RHV-BC. Therefore, the present study was conceived and aimed to investigate the clinical characteristics and prognostic factors of patients with RHV-BC in our center.

\section{Materials and Methods}

\section{Patient Inclusion}

After being approved by the Institutional Review Board, we used the database of our center to identify potential patients with RHV-BC between March 2009 and April 2019. The main clinical information, including age, gender, smoking history, and radiology were collected.

\section{Pathological Evaluation}

All cases were diagnosed by experienced pathologists who specialized in urology. Based on the 2016 WHO classification of bladder cancer, histological variants included MPV, SAV, NEV, NV, PCV, and LEV, excluding squamous variant and glandular variant. Due to different percentages of $\mathrm{VH}$ driving the same outcomes, we defined RHV-BC as any RVH appeared in specimen. ${ }^{13}$ Tumor stage, lymph node (LN) invasion, metastasis, and LVI were evaluated according to the 2016 American Joint Committee on Cancer (AJCC) TNM staging system, and tumor grade was referred to the 2016 WHO grading system. ${ }^{5,14}$
Patients were followed up either by phone or face-toface interview. All patients diagnosed with metastasis were identified by Positron Emission computed Tomography (PET-CT). OS was defined as the time from the date of surgery to death due to any cause. Recurrence-free survival (RFS) was defined as the time from the date of surgery to local or distant recurrence. Cancer-specific survival (CSS) was defined as the time from the date of surgery to the date of death from bladder cancer.

\section{Statistical Analyses}

The univariate and multivariate analysis were performed to investigate the association between potential factors and the prognosis. Kaplan-Meier analysis was employed to show the survival situation for patients with RHV-BC. All statistical analyses were processed by SPSS Version 25.0 (IBM, Armonk, NY, USA). A $P<0.05$ was considered to be statistically significant.

\section{Results}

\section{Clinical Characteristics}

A total of 3803 potential patients with bladder cancer were screened and, finally, 54 patients were identified with a rate of $1.4 \%$. Forty-four males and ten females were included with a mean age of 66.5 years old. Hematuria was detected in 49 patients ( 47 with gross hematuria and two with microscopic hematuria). Among 12 patients with metastasis, tumor was metastasized to bone $(n=5)$, lung $(\mathrm{n}=4)$, liver $(\mathrm{n}=1)$, and nasopharynx $(\mathrm{n}=1)$, while two patients had multiple metastases (one with metastases of bone, lung, and colon, the other with metastases of bone, rectus abdominis muscle, and liver). Other clinical characteristics are presented in Table 1.

\section{Management and Prognosis}

Transurethral resection of bladder tumor (TURBT) was performed among all patients for initial diagnosis or treatment. Thirty-two patients received IVT of epirubicin. Three of them only underwent immediate instillation after TURBT, while the others finished a total of 12 IVT with mild complications. RC was performed in 31 patients, and a positive surgical margin (PSM) was found in a patient with MPV-BC. Twenty-three patients received AC of GC (gemcitabine, cisplatin), six of them were diagnosed with metastasis before surgery. The number of $\mathrm{AC}$ circles depended on the response and physical status of patients. 
Table I The Clinical Characteristics of Patients with RHV-BC

\begin{tabular}{|c|c|c|c|c|c|c|c|}
\hline & Total & MPV & SAV & NEV & NV & PCV & LEV \\
\hline & $(n=54)$ & $(n=19)$ & $(n=18)$ & $(n=7)$ & $(n=6)$ & $(n=3)$ & $(n=I)$ \\
\hline Age (years, Mean $\pm S D$ ) & $66.5 \pm 13.5$ & $71.1 \pm 8.5$ & $64.8 \pm 11.9$ & $59.9 \pm 9.1$ & $67.7 \pm 14.2$ & $57.3 \pm 4.7$ & 77 \\
\hline \multicolumn{8}{|l|}{ Gender } \\
\hline Male & 44 & 17 & 13 & 6 & 4 & 3 & I \\
\hline Female & 10 & 2 & 5 & 1 & 2 & 0 & 0 \\
\hline \multicolumn{8}{|l|}{ Smoking history } \\
\hline Yes & 19 & 7 & 5 & 5 & I & I & 0 \\
\hline No & 35 & 12 & 13 & 2 & 5 & 2 & 1 \\
\hline \multicolumn{8}{|l|}{ Hematuria } \\
\hline Yes & 49 & 17 & 17 & 7 & 5 & 2 & I \\
\hline No & 5 & 2 & I & 0 & I & I & 0 \\
\hline \multicolumn{8}{|l|}{ WHO grade } \\
\hline High & 45 & 15 & 15 & 7 & 4 & 3 & I \\
\hline Low & 9 & 4 & 3 & 0 & 2 & 0 & 0 \\
\hline \multicolumn{8}{|l|}{ Tumor size* } \\
\hline$\geq 3 \mathrm{~cm}$ & 44 & 12 & 17 & 7 & 5 & 2 & I \\
\hline$<3 \mathrm{~cm}$ & 10 & 7 & I & 0 & 1 & I & 0 \\
\hline \multicolumn{8}{|l|}{ Tumor numbers } \\
\hline Multiple & 25 & 8 & 9 & 3 & 5 & 0 & 0 \\
\hline Single & 29 & 11 & 9 & 4 & 1 & 3 & I \\
\hline \multicolumn{8}{|l|}{ T stage } \\
\hline $\mathrm{T}<2$ & 32 & 12 & 10 & 5 & 2 & 2 & 0 \\
\hline $\mathrm{T} \geq 2$ & 22 & 7 & 7 & 2 & 4 & I & I \\
\hline \multicolumn{8}{|l|}{$N$ stage } \\
\hline No & 13 & 4 & 3 & I & 4 & 0 & I \\
\hline $\mathrm{N} \geq \mathrm{I}$ & 41 & 15 & 15 & 6 & 2 & 3 & 0 \\
\hline \multicolumn{8}{|l|}{ Metastasis } \\
\hline Yes & 12 & 4 & 4 & 0 & 3 & 0 & I \\
\hline No & 42 & 15 & 14 & 7 & 3 & 3 & 0 \\
\hline \multicolumn{8}{|l|}{ LVI } \\
\hline Yes & 17 & 5 & 4 & 3 & 4 & 0 & I \\
\hline No & 37 & 14 & 14 & 4 & 2 & 3 & 0 \\
\hline
\end{tabular}

Note: *For multiple tumors, the diameter of the largest tumor was regarded as tumor size.

The mean follow-up was 25.8 months with a range of 12-84 months. The mean of RFS, CSS, and OS were 18.8 months, 37.0 months, and 36.0 months, respectively. And the single Kaplan-Meier curves of RFS, CSS, and OS are displayed in supplementary Figure 1. Recurrence was detected in $81.0 \%(34 / 42)$ of patients who had not been diagnosed with metastasis at first. In detail, 27 of 32 $(84.4 \%)$ patients undergoing transurethral resection of bladder cancer (TURBT) and intravesical therapies (IVT) suffered recurrence, with a mean time to recurrence of 9.5 months. A total of 17 patients, without metastasis at initial diagnosis, received adjuvant chemotherapy (AC) after radical cystectomy (RC) and 11 of them (64.7\%) experienced recurrence, with a mean time to recurrence of 10.4 months. Other detailed information can be seen in Figure 1.

\section{Univariable and Multivariable Cox Regression Analyses Outcomes}

The univariable analysis showed that age ( $>60$ years old), $\mathrm{T} \geq 2, \mathrm{~N} \geq 1$, LVI, WHO high grade, multiple tumors, and 


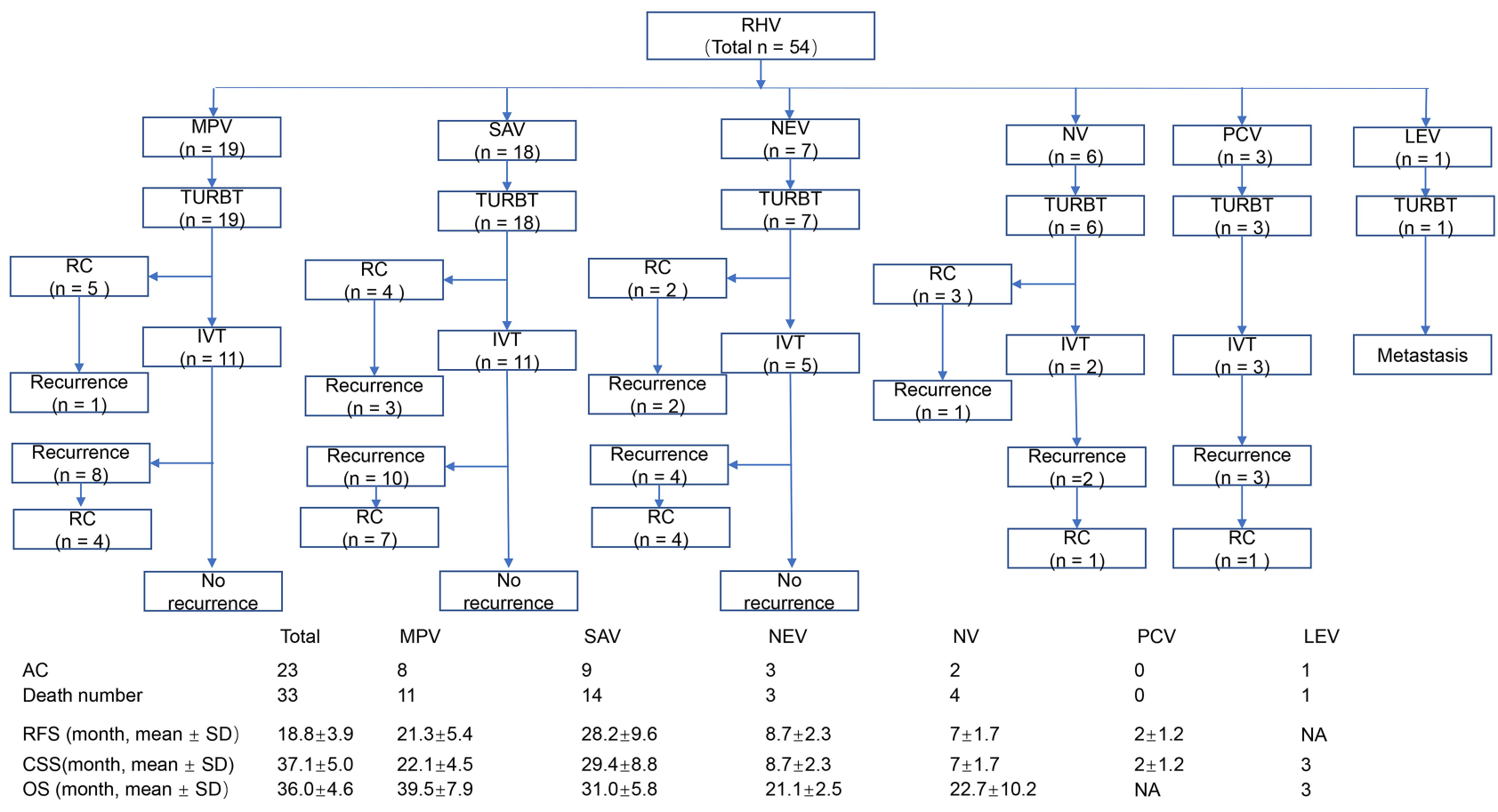

Figure I The management and prognosis of patients with RHV-BC.

metastasis were associated with worse OS for patients with RHV-BC (all $P<0.05$ ). The multivariate COX regression model indicated that metastasis (hazard ratio $[\mathrm{HR}]=2.97$, 95\% $\mathrm{CI}=1.03-8.52 ; P=0.04)$ and $\mathrm{T} \geq 2 \quad(\mathrm{HR}=3.66,95 \%$ $\mathrm{CI}=1.36-9.84 ; P=0.01)$ were independent risk factors of RHV-BC (Table 2).

\section{Discussion}

The present study reported an incidence of $1.4 \%$ of RHV$\mathrm{BC}$ in all urothelial carcinoma of bladder, which was inconsistent to the finding by Moschini et $\mathrm{al}^{6}$ with a rate of $1.6 \%$. And the largest two subtypes are MPV-BC and SAV-BC. Morphologically, MPV-BC consists of small nests and aggregates of tumor cells within lacunae without vascular cores. ${ }^{4}$ The appearance of MPV independently predicted a worse increased risk of death. ${ }^{3}$ With the feature of spindled cells, SAV-BC shows an epithelial-tomesenchymal transition from the urothelial to the sarcomatoid morphology. ${ }^{15}$ The detection of SAV-BC generally portends a poor prognosis based on a more advanced stage at diagnosis and worse OS. ${ }^{10}$

Patients with RHV-BC had a higher proportion of advanced stage and worse prognosis than those with pure bladder cancer. ${ }^{2}$ It is critical to make decisions upon the management. Although BCG instillation is recommended as the first-line treatment for bladder cancer, a low response

Table 2 Univariate and Multivariate Analysis of the Association of Different Factors with OS

\begin{tabular}{|c|c|c|c|c|c|c|}
\hline \multirow[t]{2}{*}{ Characteristics } & \multicolumn{3}{|c|}{ Univariate Analysis } & \multicolumn{3}{|c|}{ Multivariate Analysis } \\
\hline & HR & $95 \% \mathrm{Cl}$ & $P$-value & HR & $95 \% \mathrm{Cl}$ & $P$-value \\
\hline Age (>60 years old) & 0.39 & $0.18-0.87$ & 0.02 & 0.62 & $0.24-1.60$ & 0.32 \\
\hline Gender (male) & 0.98 & $0.38-2.60$ & 0.98 & - & - & - \\
\hline WHO high grade & 2.84 & I.02-7.87 & 0.05 & 1.76 & $0.56-5.53$ & 0.34 \\
\hline Smoking history & 0.82 & $0.40-1.70$ & 0.60 & - & - & - \\
\hline $\mathrm{T} \geq 2$ & 5.76 & $2.62-12.65$ & $<0.001$ & 3.66 & $1.36-9.84$ & 0.01 \\
\hline$N \geq 1$ & 7.00 & $3.14-15.64$ & $<0.001$ & 3.40 & $0.79-14.59$ & 0.10 \\
\hline Metastasis & 7.27 & $3.19-16.54$ & $<0.001$ & 2.97 & $1.03-8.52$ & 0.04 \\
\hline LVI & 7.00 & $3.14-15.64$ & $<0.001$ & 2.89 & $0.20-5.87$ & 0.87 \\
\hline Multiple tumors & 2.50 & $1.24-5.05$ & 0.01 & 1.50 & $0.68-3.30$ & 0.31 \\
\hline Tumor size $(\geq 3 \mathrm{~cm})$ & 2.69 & $0.82-8.81$ & 0.10 & - & - & - \\
\hline
\end{tabular}


rate was detected in those with MPV, SAV, and PCV-BC. ${ }^{9,16}$ Studies indicated that early RC might be a better choice for T1 MPV-BC because of a higher CSS than BCG. ${ }^{9,17}$ IVT with chemotherapeutic drugs was just reported in a few studies. ${ }^{4,12}$ Sui et $\mathrm{al}^{10}$ reported that patients with SAV-BC did not benefit from IVT following TURBT over TURBT alone. Our study also found a high recurrence rate of $84.4 \%$ among patients receiving IVT of epirubicin, which might indicate that early RC should be performed for patients with RHV-BC. Unfortunately, AC could not improve the prognosis of patients with $\mathrm{HV}$ according to the study by Berg et al. ${ }^{18} \mathrm{We}$ also found a recurrence rate of $64.7 \%$ in patients with AC. This result may be explained by gene mutation (TP53 and RB1 mutation) and RNA expression (lnRNA LBCS, miR-296), which were identified in RHV-BC and associated with chemoresistance ${ }^{19-21}$ Besides, although neoadjuvant chemotherapy (NAC) was recommended for eligible patients with muscle invasive bladder cancer, it could not benefit the prognosis of MPV and SAV., ${ }^{9,10}$ And no patients received NAC in the present study.

On the other hand, many studies attempted to find critical risk factors of prognosis. Age, female, American Society of Anesthesiologists (ASA) score, T stage, LVI, $\mathrm{N}$ stage, and metastasis were identified as independent predictors of patients with pure bladder cancer. ${ }^{22,23}$ In this study, metastasis was detected in 12 patients when initially diagnosed with RVH-BC and was confirmed as an independent risk factor of OS, which was consistent with the study by Lobo et al. ${ }^{24}$ One 34-year-old patient was found with SAV-BC and multiple metastases in bone, colon, and lung by PET-CT, and died within 3 months. Muscle invasive bladder cancer (MIBC) or $\mathrm{T} \geq 2$ was regarded as a predictor of worse prognosis than NMIBC, and the indication for performing RC. ${ }^{13,25}$ For patients with RHV-BC, $\mathrm{T} \geq 2$ was related to a shorter OS based on the univariable and multivariable analysis. Besides, it was found that LN invasion was associated with a high recurrence rate and low survival rate for patients with pure bladder cancer. ${ }^{26}$ As for HV-BC, Koguchi et $\mathrm{al}^{2}$ reported that lymph node (LN) invasion was an independent risk factor of worse OS. And the LN invasion was related with the alteration of RHV-BC in gene (TP53 and RB1 mutation), RNA expression (DANCR, Ki67 mRNA), and cellular factors (VEGF-C, CK7). ${ }^{21,27,28}$ We had the same conclusion among patients with RHV-BC, but only on the basis of univariable analysis, not multivariable analysis. This was probably due to a limited sample size. LVI was an important index in the pathological evaluation and was related to worse prognosis in patients with pure bladder cancer. ${ }^{29}$ But our study did not find a significant result in RHV-BC. Several studies reported LVI was commonly observed in MPV-BC with advanced $\mathrm{T}$ stages and it was associated with poor prognosis, while none of these studies indicated LVI as an independent risk factor of OS according to multivariable analysis. ${ }^{12,30,31}$ Similarly, a study enrolling 729 cases with HV did not show that LVI was an independent risk factor of overall mortality. ${ }^{6}$ It was found that tumor grade did not influence the prognosis of MPV-BC according to the study by Vourganti et al. ${ }^{32}$ In the present study, the multivariable analysis indicated that high and low grade tumors had the same OS in patients with RHV-BC. It is noticed that all RHV-BC should be managed as high grade tumors regardless of tumor grade, which was consistent with the guidelines of the European Association of Urology (EAU). ${ }^{33}$

There are several limitations in the present study. First, this is a single-center, retrospective study based on a hospital information system. The bias of data might be noticed. Second, because of the limited sample size, especially some subtypes of RHV-BC, no comparison among different subtypes could be performed. Besides, the effect of management was not included, mainly due to the variety of groups. However, detailed information was collected and presented to provide a comprehensive view on the principle characteristics of RHV-BC.

\section{Conclusion}

With a low incidence, RHV was likely seen in elder patients. MPV and SAV were the most common two subtypes of RHV. $T \geq 2$ and metastasis were independent risk factors of OS in patients with RHV-BC according to multivariable analyses. Considering a high recurrence following TURBT and IVT, early radical cystectomy might be performed for patients with RHV-BC. More studies with larger sample sizes are called for providing more higher level of evidence.

\section{Abbreviations}

RHV-BC, rare histological variants of bladder cancer; MPV, micropapillary variant; SAV, sarcomatoid variant; $\mathrm{NEV}$, neuroendocrine variant; NV, nested variant; PCV, plasmacytoid variant; LEV, lymphoepithelioma-like variant; AC, adjuvant chemotherapy; CI, confidence interval; CSS, cancer-specific survival; DANCR, differentiation antagonizing non-protein coding RNA; RC, radical cystectomy; RFS, recurrence-free survival; HR, hazard ratio; IVT, intravesical therapies; lncRNA, long noncoding RNAs; LVI, lymphovascular invasion; NA, no applicable; 
OS, overall survival; SD, standard deviation; TURBT, transurethral resection of bladder tumor; VEGF-C, vascular endothelial growth factor $\mathrm{C}$; WHO, World Health Organization.

\section{Ethics and Consent Statement}

The study and disclaimer of informed consent were approved by the West China Hospital of Sichuan University Biomedical Research Ethics Committee (No. 2020366). Moreover, all these procedures were performed in accordance with the ethical standards established in the Declaration of Helsinki. All patients provided written informed consent before starting the study.

\section{Author Contributions}

All authors made substantial contributions to the conception and design, acquisition of data, or analysis and interpretation of data; took part in drafting the article or revising it critically for important intellectual content; agreed to submit to the current journal; gave final approval of the version to be published; and agree to be accountable for all aspects of the work.

\section{Funding}

The study was supported by the Pillar Program from Department of Science and Technology of Sichuan Province (2018SZ0219) and the 1.3.5 project for disciplines of excellence, West China Hospital, Sichuan University (ZY2016104).

\section{Disclosure}

The authors report no conflicts of interest for this work.

\section{References}

1. Freddie B, Jacques F, Isabelle S, et al. Global cancer statistics 2018: globocan estimates of incidence and mortality worldwide for 36 cancers in 185 countries, Cancer. J Clin. 2018;68(6):394-424. doi: $10.3322 /$ caac. 21492

2. Koguchi D, Matsumoto K, Ikeda M, et al. Histologic variants associated with biological aggressiveness and poor prognosis in patients treated with radical cystectomy. Jpn J Clin Oncol. 2019;49 (4):373-378. doi:10.1093/jjco/hyz015

3. Sui W, Matulay JT, James MB, et al. Micropapillary bladder cancer: insights from the national cancer database. Bladder Cancer. 2016;2 (4):415-423. doi:10.3233/BLC-160066

4. Moschini M, D'Andrea D, Korn S, et al. Characteristics and clinical significance of histological variants of bladder cancer. Nat Rev Urol. 2017;14(11):651-668. doi:10.1038/nrurol.2017.125

5. Humphrey PA, Moch H, Cubilla AL, et al. The 2016 WHO classification of tumours of the urinary system and male genital organs-part b: prostate and bladder tumours. Eur Urol. 2016;70(1):106-119. doi:10.1016/j.eururo.2016.02.028
6. Moschini M, Dell'Oglio P, Luciano R, et al. and effect of variant histology on oncological outcomes in patients with bladder cancer treated with radical cystectomy. Urol Oncol. 2017;35(6):335-341. doi:10.1016/j.urolonc.2016.12.006

7. Oddens J, Sylvester R, Brausi M, et al. The effect of age on the efficacy of maintenance Bacillus Calmette-Guérin relative to maintenance epirubicin in patients with stage $\mathrm{Ta} \mathrm{T} 1$ urothelial bladder cancer: results from EORTC genito-urinary group study 30911. Eur Urol. 2014;66(4):694-701. doi:10.1016/j.eururo.2014.05.033

8. Arends TJ, Nativ O, Maffezzini M, et al. of a randomised controlled trial comparing intravesical chemohyperthermia with mitomycin c versus bacillus calmette-guerin for adjuvant treatment of patients with intermediate- and high-risk non-muscle-invasive bladder cancer. Eur Urol. 2016;69(6):1046-1052. doi:10.1016/j.eururo.2016.01.006

9. Abufaraj M, Foerster B, Schernhammer E, et al. urothelial carcinoma of the bladder: a systematic review and meta-analysis of disease characteristics and treatment outcomes. Eur Urol. 2019;75 (4):649-658. doi:10.1016/j.eururo.2018.11.052

10. Sui W, Matulay JT, Onyeji IC, et al. treatment patterns and outcomes of sarcomatoid bladder cancer. World J Urol. 2017;35(7):1055-1061. doi:10.1007/s00345-016-1962-8

11. Li Z, Liao H, Tan Z, et al. bladder cancer: a clinico-pathological characterization and treatment analysis. Clin Transl Oncol. 2017;19 (10):1217-1224. doi:10.1007/s12094-017-1658-6

12. Wang JK, Boorjian SA, Cheville JC, et al. following radical cystectomy for micropapillary bladder cancer versus pure urothelial carcinoma: a matched cohort analysis. World J Urol. 2012;30(6):801-806. doi:10.1007/s00345-012-0976-0

13. Kaimakliotis HZ, Monn MF, Cary KC, et al. Plasmacytoid variant urothelial bladder cancer: is it time to update the treatment paradigm? Urol Oncol. 2014;32(6):833-838. doi:10.1016/j.urolonc.2014.03.008

14. Amin MB. AJCC Cancer Staging Manual 8th Edition. 2016;105 (10):1402-1412.

15. Genitsch V, Kolloa A, Vandekerkhove G, et al. Morphologic and genomic characterization of urothelial to sarcomatoid transition in muscle-invasive bladder cancer. Urol Oncol. 2019;37(9):e19-e29. doi:10.1016/j.urolonc.2019.06.021

16. Porten SP, Willis D, Kamat AM. Variant histology: role in management and prognosis of nonmuscle invasive bladder cancer. Curr OpinUro. 2014;124(5):517-523. doi:10.1097/MOU.0000000000000089

17. Kamat AM, Gee JR, Dinney CPN, et al. The case for early cystectomy in the treatment of nonmuscle invasive micropapillary bladder carcinoma. J Uro. 2006;175(3Pt1):881-885. doi:10.1016/S00225347(05)00423-4

18. Berg S, D'Andrea D, Vetterlein MW, et al. of adjuvant chemotherapy in patients with adverse features and variant histology at radical cystectomy for muscle-invasive carcinoma of the bladder: does histologic subtype matter? Cancer. 2019;125(9):1449-1458. doi:10.1002/cncr.31952

19. Hikmat A, George J. Updates on the Genomics of Bladder Cancer and Novel Molecular Taxonomy. Adv Anat Pathol. 2020;27 (1):36-43. doi:10.1097/PAP.0000000000000252

20. Chen X, Xie R, Gu P, et al. Long Noncoding RNA LBCS Inhibits Self-Renewal and Chemoresistance of Bladder Cancer Stem Cells through Epigenetic Silencing of SOX2. Clin Cancer Res. 2019;25 (4):1389-1403. doi:10.1158/1078-0432.CCR-18-1656

21. Robertson AG, Kim J, Al-Ahmadie H, et al. Comprehensive Molecular Characterization of Muscle-Invasive Bladder Cancer. Cell. 2017;171(3):540-556e525. doi:10.1016/j.cell.2017.09.007

22. Del Pozo Jiménez G, Herranz Amo F, Díez Cordero JM, et al. Prognostic factors of mortality in patients with urothelial bladder tumor treated with radical cistectomy. Arch Esp Urol. 2019;72 (5):451-462.

23. Quek M, Stein J, Nichols P, et al. significance of lymphovascular invasion of bladder cancer treated with radical cystectomy. $J$ Uro. 2014;174(1):103-106. doi:10.1097/01.ju.0000163267.93769.d8 
24. Lobo N, Shariat SF, Guo CC, et al. What Is the Significance of Variant Histology in Urothelial Carcinoma? Eur Urol Focus. 2019;794:0-11. doi:10.1016/j.euf.2019.09.003

25. Stein JP, Lieskovsky G, Cote R, et al. Cystectomy in the treatment of invasive bladder cancer: long-term results in 1054 patients. $J$ Clin Oncol. 2001;19(9):666-675. doi:10.1200/JCO.2001.19. 3.666

26. Cha EK, Sfakianos JP, Sukhu R, et al. Poor prognosis of bladder cancer patients with occult lymph node metastases treated with neoadjuvant chemotherapy. BJU Int. 2018;122(4):627-632. doi:10.1111/bju.14242

27. Wang C, Liu Q, Huang M, et al. Loss of GATA6 expression promotes lymphatic metastasis in bladder cancer. FASEB J. 2020;34 (4):5754-5766. doi:10.1096/fj.201903176R

28. Chen Z, Chen X, Xie R, et al. DANCR Promotes Metastasis and Proliferation in Bladder Cancer Cells by Enhancing IL-11-STAT3 Signaling and CCND1 Expression. Mol Ther. 2019;27(2):326-341. doi:10.1016/j.ymthe.2018.12.015
29. Mari A, Kimura S, Foerster B, Abufaraj M. A systematic review and meta-analysis of lymphovascular invasion in patients treated with radical cystectomy for bladder cancer. Urol Oncol. 2018; S1078143918301145. doi:10.1016/j.urolonc.2018.03.018

30. Amin, Mahul B. Histological variants of urothelial carcinoma: diagnostic, therapeutic and prognostic implications. Mod Pathol. 2009;22. doi:10.1038/modpathol.2009.26

31. Kaimakliotis HZ, Monn MF, Cheng L, et al. Plasmacytoid bladder cancer: variant histology with aggressive behavior and a new mode of invasion along fascial planes. Urology. 2014;83(5):1112-1116. doi:10.1016/j.urology.2013.12.035

32. Vourganti S, Harbin A, Singer EA, et al. Grade Micropapillary Urothelial Carcinoma, Does It Exist? - Analysis of Management and Outcomes from the Surveillance, Epidemiology and End Results (SEER) Database. J Cancer. 2013;4(4):336-342. doi:10.7150/jca.6215

33. EAU Guidelines. Edn. presented at the EAU Annual Congress Amsterdam. ISBN 978-94-92671-07-3. 2020.

\section{Publish your work in this journal}

Cancer Management and Research is an international, peer-reviewed open access journal focusing on cancer research and the optimal use of preventative and integrated treatment interventions to achieve improved outcomes, enhanced survival and quality of life for the cancer patient.
The manuscript management system is completely online and includes a very quick and fair peer-review system, which is all easy to use. Visit http://www.dovepress.com/testimonials.php to read real quotes from published authors. 